\title{
Generalized Direction Changing Fall Control of Humanoid Robots Among Multiple Objects
}

\author{
Umashankar Nagarajan and Ambarish Goswami
}

\begin{abstract}
Humanoid robots are expected to share human environments in the future and it is important to ensure safety of their operation. A serious threat to safety is the fall of a humanoid robot, which can seriously damage both the robot and objects in its surrounding. This paper proposes a strategy for planning and control of fall. The controller's objective is to prevent the robot from hitting surrounding objects during a fall by modifying its default fall direction.

We have earlier presented such a direction-changing fall controller in [1]. However, the controller was applicable only when the robot's surrounding contained a single object. In this paper we introduce a generalized approach to humanoid falldirection control among multiple objects. This new framework algorithmically establishes a desired fall direction through assigned scores, considers a number of control options, and selects and executes the best strategy. The fall planner is also able to select "No Action" as the best strategy, if appropriate. The controller is interactive and is applicable for fall occurring during upright standing or walking. The fall performance is continuously tracked and can be improved in real-time. The planning and control algorithms are demonstrated in simulation on an ASIMO-like humanoid robot.
\end{abstract}

\section{Motivation}

Although the loss of balance and fall are rare for a humanoid robot in typical controlled environments, it will be inevitable in physically interactive environments. The result of a fall can be severe both to the robot and to objects and people in the vicinity.

One can ignore the possibility of a fall and wishfully hope that its effects will not be serious. However, failure studies, such as in car crash, have taught us against behaving according to this instinct. In fact, planning and simulation of failure situations can have enormous benefits, including system design improvements, and support for user safety and confidence. Following this philosophy we closely focus our attention to the phenomenon of humanoid fall and attempt to develop a comprehensive control strategy to deal with this undesired and traumatic "failure" event.

Time is a premium during the occurrence of a fall; a single rigid body model of the Honda ASIMO robot indicates that a fall from the vertical upright stationary configuration due to a mild push takes about $800-900 \mathrm{~ms}$. In some situations the time to fall can be even shorter, and there is no opportunity for elaborate planning or time-consuming control. Yet, simulation and experimental results indicate that meaningful

This work was supported by Honda Research Institute USA

U. Nagarajan is with The Robotics Institute, Carnegie Mellon University, Pittsburgh, PA 15213, USA umashankar@cmu.edu

A. Goswami is with Honda Research Institute, Mountain View, CA 94041, USA agoswami@honda-ri. com modification to the default fall behavior can be imparted to minimize damage to the robot or to the environment.

A humanoid fall controller may have two primary, and distinctly different, goals: a) self-damage minimization and b) minimization of damage to others. When fall occurs in an open space, a self-damage minimization strategy can reduce the harmful effects of the ground impact. If, however, the falling robot can damage nearby objects or injure persons, the primary objective is to prevent this from happening. This we try to achieve by means of changing the default fall direction of the robot such that even during falling it makes no contact with the surrounding objects.

The first reported work on humanoid fall direction change was in [1] and in the current paper we present a thorough generalization, extension and improvement of this approach. A sample of our current results is shown in Fig. 1. When pushed with a large force, the humanoid robot falls on an object when no controller is active, Fig. 1(a). When the proposed fall controller is turned on, the robot successfully avoids falling on the objects, Fig. 1(b).

The fall direction change controller exploits the fact that regardless of its complex motion, a falling humanoid can topple only about an edge or a vertex of its support base. We appropriately change the robot's support base geometry to modify the position and orientation of this leading tipping edge. This profoundly influences the robot's fall behavior as it is guided away towards a free area. The support base geometry is modified through the lifting of a foot or a stepping action, and the specific parameters for these actions are selected using a brute-force process. Additional improvements are achieved using inertia shaping [2] techniques.

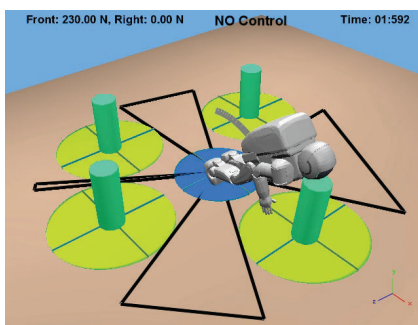

(a)

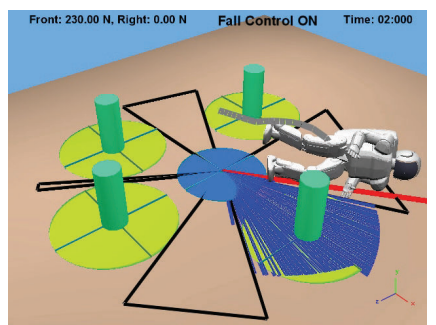

(b)
Fig. 1. A forward force of $230 \mathrm{~N}$ applied to the robot. With no control, the robot falls on an object, (a), and successfully avoids the objects when fall controller is turned on, (b). The black-outlined conical regions on the ground represent the safe fall regions. The blue lines on the ground represent the scores for each foot placement strategy evaluated and the red line gives the desired fall direction. The center of mass (CoM) trajectory during fall is shown in gray. 
There are several major improvements compared to our earlier work, as follows:

a) Multiple Objects: During a fall among multiple objects, the robot must try to avoid falling in several directions. In this paper, we newly formulate the fall direction control as a minimization problem, wherein the robot tries to minimize its deviation from a desired fall direction. The robot algorithmically determines the desired fall direction from the location of obstacles and the impending fall direction. With only one object the robot had only one fall direction to avoid, attempting to fall as far as possible from this direction (a maximization problem). See Sec. IV-A.

b) Control Trigger: As described in [1] the Fall Trigger Boundary (FTB) of a robot encloses a region in the robot's state space in which a given balance controller is able to stabilize the robot. An exit through the FTB is an indication of a certain fall and this event was used to activate a switch from the robot's balance controller to a fall controller. In our current work, the fall trigger, which is the earliest prediction of a fall, is distinguished from the control trigger, when the control is launched. The controller must wait till the control trigger because fall trigger may occur too early for the robot to possess sufficient information to make an intelligent decision. See Sec. III-D.

c) Simultaneous Foot Placement and Inertia Shaping: The inertia shaping technique [2] to control the overall composite rigid body (CRB) inertia [3] of the robot cannot be launched before foot placement is completed, because the two actions may be in conflict. Therefore, we introduce partial inertia shaping, which is a procedure to change the CRB inertia of the robot simultaneously during foot placement, without using the joints involved in the latter. See Sec. IV-B.

d) Inertia Shaping about CoP: The inertia shaping procedure is used to change the CRB inertia of the robot. In this paper we perform inertia shaping at the center of pressure $(\mathrm{CoP})$ and not at the $\mathrm{CoM}$, for reasons discussed in Sec. IV-B.

\section{RELATED WORK}

A number of recent papers reported on the damage minimization aspect of humanoid fall. In their exhaustive work Fujiwara et al. ([4], [5], [6], [7], [8]) proposed martial arts type motion for damage reduction, computed optimal falling motions using minimum impact and angular momentum, and fabricated special hardware for fall damage study. Ogata et al. proposed [9], [10] two fall detection methods based on abnormality detection and predicted ZMP. The robot improves fall detection through exponential learning or through online CoM trajectory calculation. Renner and Behnke[11] use model-based approach to detect external forces on the robot and Daniel Karssen and Wisse[12] use principal component analysis to detect fall. Following human movement based search procedure Ruiz-del-Solar et al. implemented a low damage fall sequence for soccer robots[13]. In [14], [15] fall detection and control are treated together using Gaussian mixture models and Hidden Markov model. Ishida et al. employed servo loop gain shift to reduce shock due to fall[16]. Fall damage minimization is obviously of natural interest in biomechanics[17].

\section{KEY CONCEPTS}

This section will describe some of the key concepts used throughout the paper.

\section{A. Geometric Setup}

In $3-\mathrm{D}$, both the robot and the surrounding objects are approximated by circumscribing vertical cylinders centered at their respective CoMs. On the horizontal projection, the objects are represented by circles and the robot is represented by a circle with its center at the CoM and the maximum leg spread as its diameter (Fig. 2(a)). We assume that the position and size of the objects are known to the robot at all times. Following the configuration space formulation used in traditional motion planning algorithms [18], the object circles are grown by the radius of the robot circle and the robot is reduced to a point (Fig. 2(b)). The entire planning process uses information in polar coordinates $(r, \theta)$ with the point robot at the origin $(0,0)$, where $r \in \mathbb{R}^{+}$represents the distance from the point robot and $\theta \in \Theta=[0,2 \pi]$ represents the direction. The direction $\theta=0$ represents the reference direction with respect to which all objects' positions and orientations are known. Only objects within a radius of 1.5 times the height of the robot are considered for the planning process and the other objects are considered too far from the robot to be hit.

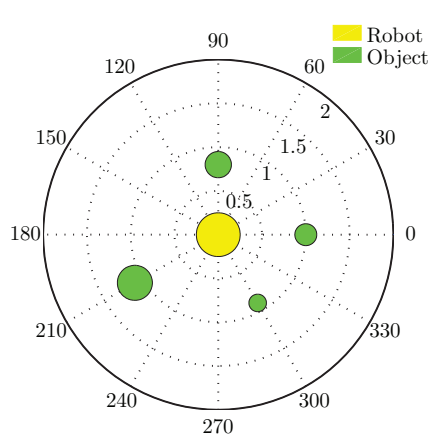

(a)

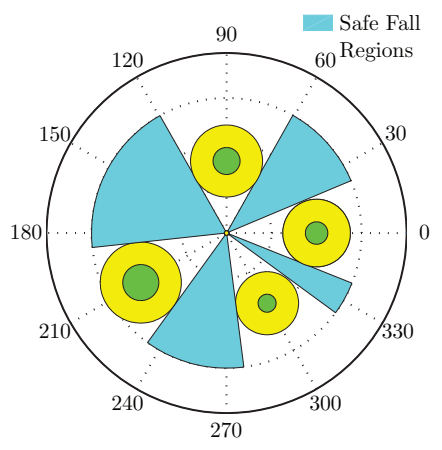

(b)
Fig. 2. The 2-D projection. (a) The yellow circle represents the robot. Its center is located at the robot's CoM and its diameter is equal to robot's maximum leg spread. The object circles shown in green are the circumscribing circles of the objects' 2-D projections. (b) The object circles are grown by robot's radius and the robot reduces to a point. Safe fall regions (cyan shaded cones) are the free cones in which the robot can fall without hitting an object.

\section{B. Fall Direction}

In this paper, fall direction is defined as the vector connecting the robot's initial and final CoM ground projections. The initial state is at control trigger and the final state is the ground robot touchdown, estimated using inverted pendulum simulations. At fall trigger, all controllers on the robot are assumed to be stopped and the joints are locked with the robot behaving like a rigid body until control trigger is reached. 
After control trigger is hit, the only active controller is the safe fall controller that is described in Sec. IV-B. The fall direction is independent of the intermediate configurations of the robot, which implies that it is independent of the CoM positions during fall. In the geometric setup described in Sec. III-A, the fall direction is given by an angle $\theta_{f} \in \Theta$.

\section{Safe Fall Regions}

A safe fall region, characterized by an object-free cone, is the set of continuous fall directions $\left(\theta_{f}\right)$ with no objects inside them as depicted by cyan cones in Fig. 2(b). These represent the set of directions in which the robot can fall without hitting an object. The number of safe fall regions, $n_{s f}$, is given by:

$$
n_{s f}=n_{o b j}-n_{\text {int }}
$$

where, $n_{o b j}$ is the number of non-intersecting objects and $n_{\text {int }}$ is the number of pairs of intersecting expanded objects. The set of all safe fall regions is given by $S F=\left\{S F_{1}, \ldots, S F_{n_{s f}}\right\}$, where $S F_{j}$ represents the $j^{t h}$ safe fall region (free cone).

\section{Fall Trigger Vs Control Trigger}

The planning and strategy evaluation procedure discussed in [1] was done at the occurrence of fall trigger. Fall trigger is set off by a fall predictor and represents the earliest warning of an impending fall. Although the fall predictor may predict the imminence of a fall very early, we might not, at that point, have sufficient information to select the best controller.

For example, the fall controller assumes that the estimation of fall direction is accurate. This assumption holds only for a steady fall, where the initial and terminal fall directions coincide. For other cases, where the fall direction evolves with time, this assumption may not hold. Therefore, in order to make the best controller selection and launch the controller, we must first ensure that the robot's tipping motion is steady. Control trigger is the instant, simultaneous or subsequent to the fall trigger, when the robot's tipping motion is ascertained to be steady.

We have used the Capture Point[19] trajectory to evaluate the steadiness of the tipping motion. The capture point is a point on the ground to which the biped, when subjected to a perturbation, can step to and stop without requiring another step. Capture point is estimated using a linear inverted pendulum model and is directly proportional to the velocity of CoM. During steady fall, the capture point trajectory is approximately a straight line (Fig. 3(a)). But, when there is a spinning motion about an axis normal to the ground, the fall is no longer steady and the capture point trajectory changes direction as shown in Fig. 3(b).

It is generally true that the longer we wait following the fall trigger, the better is our estimate of all quantities pertaining to the fall. Although a moment before the robot touches the ground, we can predict the fall direction with $100 \%$ accuracy, there is no time to do anything useful. So, there is a trade-off between information and time.

At every instant we evaluate the steadiness of a fall by computing the collinearity of a set of past capture points

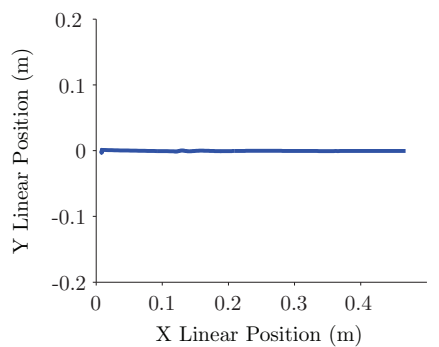

(a)

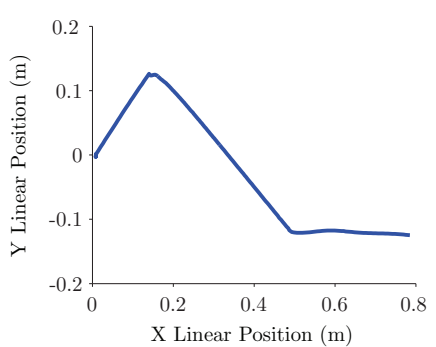

(b)
Fig. 3. Capture point trajectories of a falling humanoid. Steady tipping results in a straight line trajectory (a), and unsteady tipping motion results in a multi-segmented line (b). The straight line segments in (b) indicate steady tipping about an edge even though the leading edge may change.

ending at the current time. If steadiness is not reached within a time limit, the control trigger is automatically triggered and the fall controller immediately launched. The number of sequential capture points considered, the allowable limits of collinearity and the time limit are all hand tuned for better performance.

\section{SAFe Direction Changing Fall}

The fall direction change problem can be divided into two phases: planning and control. The planning phase consists of intelligent strategy selection and determination of a desired safe fall direction. The robot's current and predicted states and the safe fall regions are taken into account during this stage. The control phase consists of execution of the chosen strategy. This section discusses these two phases in detail.

\section{A. Planning}

1) Scoring Fall Directions: Each fall direction $\theta_{f_{i}}$ receives two scores $\left(s_{1}^{i}, s_{2}^{i}\right)$, whose weighted sum gives the total score $s^{i}$ as shown below:

$$
\begin{gathered}
s_{1}^{i}= \begin{cases}1-\frac{\Delta \theta_{f_{j}}}{\Delta \theta_{f_{\max }}}, & \text { if } \theta_{f_{i}} \in S F_{j} \\
1, & \text { if } \theta_{f_{i}} \notin S F\end{cases} \\
s_{2}^{i}= \begin{cases}\frac{2\left|\theta_{f_{i}}-\theta_{f_{j}}^{b}\right|}{\Delta \theta_{f_{j}}}, & \text { if } \theta_{f_{i}} \in S F_{j} \\
\frac{2\left|\theta_{f_{i}}-\theta_{f_{j^{*}}}^{b}\right|}{\Delta \theta_{f^{*}}}, & \text { if } \theta_{f_{i}} \notin S F \text { and } \theta_{f_{i}} \rightarrow S F_{j^{*}}\end{cases} \\
s^{i}=w_{1} s_{1}^{i}+w_{2} s_{2}^{i}, \quad w_{1}+w_{2}=1
\end{gathered}
$$

where, $\Delta \theta_{f_{j}}$ represents angle subtended by the $j^{\text {th }}$ safe fall region $S F_{j}, \theta_{f_{j}}^{b}$ represents the absolute angle of the bisector of the $j^{\text {th }}$ safe fall region, $\Delta \theta_{f_{\max }}$ represents the angle subtended by the largest safe fall region and $\theta_{f_{i}} \rightarrow S F_{j^{*}}$ means that the $j^{\text {th }}$ safe fall region is the closest to $\theta_{f_{i}}$. It is to be noted that when $\theta_{f_{i}} \in S F, s_{1}^{i} \in[0,1], s_{2}^{i} \in[0,1], s^{i} \in[0,1]$ and when $\theta_{f_{i}} \notin S F, s_{1}^{i}=1, s_{2}^{i}>1, s^{i}>1$. i.e. the safe fall directions receive a score less than or equal to one, whereas the unsafe fall directions receive a score greater than one. The total score $s^{i}$ is zero when the fall direction $\theta_{i}$ is at the bisector of the largest safe fall region. Therefore, lower the score, safer is the fall direction. 
2) Foot Placement Strategies: The planner evaluates and selects from three foot placement strategies: a) No Action, b)Lift a Leg and c)Take a Step.

a) No Action: There is no attempt at controlling the robot beyond locking all joints and letting the robot fall down as a rigid body. This strategy is adopted when the default fall direction of the robot is already deemed safe.

b) Lift a Leg: This strategy is evaluated only when the robot is in double-support phase. It involves two substrategies, 1)lift left leg and 2)lift right leg. Lifting a leg reduces the extent of support base to a single footprint. Although apparently simple, this strategy can exert significant influence on the toppling motion.

c) Take a Step: This strategy involves taking a step from the robot's current position. The number of possible stepping locations provides a number of sub-strategies to be evaluated. An inverted pendulum model is used to estimate the amount of time available before the robot touches the ground. This is used as the control duration for estimating the allowable stepping region with leg Jacobians[1].

An appropriately taken step changes the support base geometry the most and can exert a powerful nudge to the robot's fall direction. However, it also takes a relatively long time to complete the step, and if the robot is already undergoing tipping, there might not be enough time to complete the step. There are cases where the swing foot touches the ground before the step completion due to severe inclination of the robot.

3) Inertia Shaping Strategies: Inertia shaping (Sec. IVB.2) strategies are sometimes used in conjunction with, and at other times as a replacement for, the foot placement strategies.

a) Whole Body Inertia Shaping: This strategy involves employing inertia shaping techniques on the entire robot. This technique recruits all the joints and replaces the foot placement strategy when it fails to produce a safe fall.

b) Partial Inertia Shaping: This strategy involves using inertia shaping techniques only on those joints on the humanoid that are not involved in the stepping.

4) Strategy Selection: The strategy selection is done in a logical manner as presented in Fig. 4.

In case of steady fall, the fall direction estimation is more accurate and the No Action and Lift a Leg strategies are given preference over Take a Step strategies because the former are guaranteed for a successful completion. In case of unsteady fall or when the No Action and Lift a Leg strategies fail to produce safe fall, all foot placement strategies are evaluated and their estimated fall directions are assigned scores. The strategy with the minimum total score is chosen to be the optimal safe fall direction. As one can see, even when no foot placement strategy produces a safe fall direction, the algorithm chooses the strategy with the lowest score that corresponds to the fall direction closest to the safe fall region.

When no foot placement strategy produces safe fall, partial inertia shaping strategy is coupled with the optimal foot placement strategy. The bisector of the safe fall region closest to the direction corresponding to the optimal foot placement

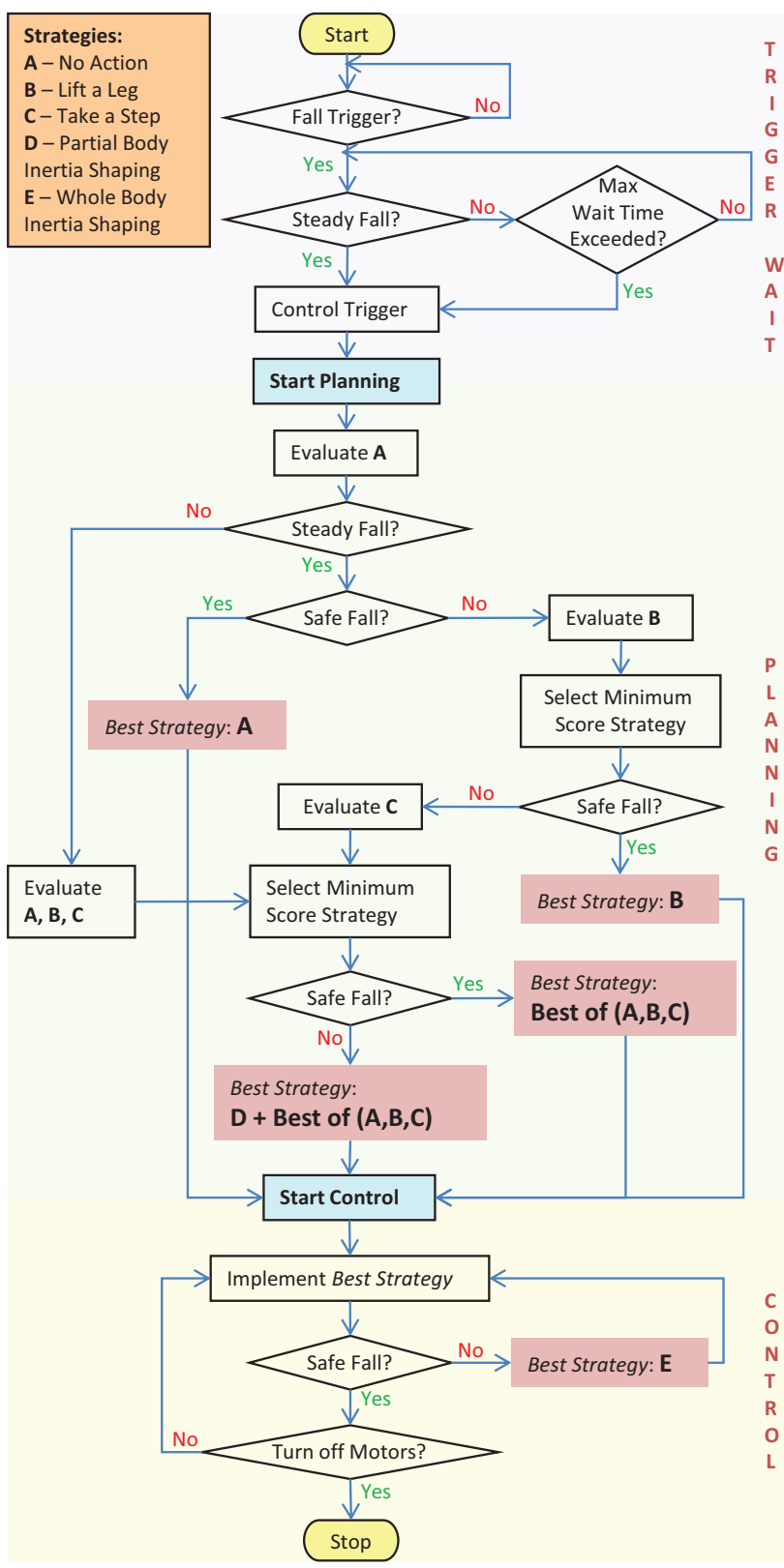

Fig. 4. Decision making procedure for safe fall planning and control.

strategy is chosen to be the desired direction for the partial inertia shaping procedure. This fall direction corresponds to the local minima closest to the current fall direction. While the optimal foot placement strategy tries to do the best it can, partial inertia shaping tries to move the body to the closest safe fall region.

The strategy selection procedure described above happens only at control trigger. The strategy execution described in Sec. IV-B happens after control trigger as shown in Fig. 4. At any future time after execution of the chosen strategy, if the robot's fall direction is unsafe, whole body inertia shaping is initiated. The bisector of the safe fall region closest to the current fall direction is chosen to be the desired direction of fall and the inertia shaping procedure tries to achieve it.

Finally, if the robot's inclination angle exceeds a maximum 
threshold, all the motors are turned off, i.e. all joints are unlocked, in order to reduce damage due to impact.

\section{B. Control}

This section describes the implementation of stepping and inertia shaping strategies.

1) Stepping Control: Given a desired stepping location, we can achieve it by controlling the leg joints through inverse kinematics. However, precise stepping is not trivial in case of a tipping robot. The robot behaves as an underactuated system and based on its joint angles, the robot does not know that it is tipping. The only way the robot would know this is with the use of other sensors like a gyroscope. The Honda ASIMO robot has a 3-axis gyroscope and 3-axis accelerometer, which can provide the information needed to compute the base frame's tipping transformation. The base frame is attached to the centroid of the trunk. The controller uses Jacobians to control the stepping motion. The necessary joint velocities $\dot{\theta}$ to move the swing leg to the desired location is given by:

$$
\dot{\theta}=J_{R}^{L \#}\left(\dot{P}_{R}-\dot{P}_{L}\right)
$$

where, $J_{R}^{L^{\#}}$ is the pseudo-inverse of foot-to-foot Jacobian $J_{R}^{L}$, $\dot{P}_{R}$ and $\dot{P}_{L}$ are the linear velocities of the right and left feet respectively.

2) Inertia Shaping Control: If the foot placement strategy fails to produce a safe fall, the robot can attempt to change the fall direction using inertia shaping [2]. This technique changes the fall direction by generating an angular momentum in the desired direction of fall. In inertia shaping we control the CRB inertia of the robot.

The inertia shaping control described here is used to move the robot in a desired direction, whereas in [1] it was used to move the robot away from the direction to be avoided. Details of deriving the desired inertia matrix $I_{d}$ can be found in [1].

Here inertia shaping is performed about the $\mathrm{CoP}\left(I^{P}\right)$ and not about the CoM $\left(I^{G}\right)$ as in [1]. This makes sense beause the desired angular velocity used to derive the desired inertia is computed about the CoP frame and hence the inertia shaping procedure should also be done about the same frame. Moreover, partial inertia shaping is more effective about CoP. This is because the arm configurations make more significant contributions to the CRB inertia about CoP. So, the desired inertia matrix $I_{d}$ derived here is about CoP i.e. $I_{d}^{P}$ (Fig. 5).

To implement inertia shaping, we string out the 6 unique elements of the CRB inertia matrix in the form of a vector: $I_{(3 \times 3)} \rightarrow \hat{I}_{(6 \times 1)}$. Next we obtain the CRB inertia Jacobian $J_{I}$ which maps changes in the robot joint angles into corresponding changes in $\hat{I}$, i.e., $\delta \hat{I}=J_{I} \delta \theta$. The desired joint velocities to attain $I_{d}$ are given by:

$$
\dot{\theta}=J_{I}^{\#}\left(\hat{I}_{d}-\hat{I}\right)
$$

where $J_{I}^{\#}$ is the pseudo-inverse of $J_{I}$.

Eq. 6 is used for whole body inertia shaping. During partial inertia shaping, we recruit only the upper body joints

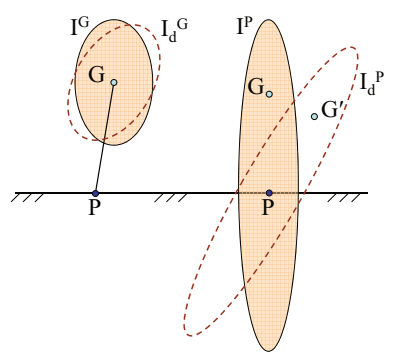

Fig. 5. Comparing inertia shaping about $\mathrm{CoM}$ (left) and $\mathrm{CoP}$ (right). Ellipsoids with solid and shaded outlines, in each case, denotes current and desired inertia, respectively. Note that inertia shaping about the CoP allows a movement of the CoM $\left(G\right.$ to $\left.G^{\prime}\right)$, which the other does not.

for Take a Step and stance leg joints for Lift a Leg. The CRB inertia Jacobian $J_{I}$ can be re-written as:

$$
J_{I}=\left[\begin{array}{ll}
J_{P I S} & J_{F P}
\end{array}\right]
$$

where, $J_{P I S}$ is the CRB inertia Jacobian corresponding to the joints that are free from foot placement strategy execution, whereas $J_{F P}$ is the CRB inertia Jacobian corresponding to the joints involved in foot placement strategy execution. The desired angular velocities to attain $I_{d}$ by partial inertia shaping are given by:

$$
\dot{\theta}_{P I S}=J_{P I S}^{\#}\left(I_{d}-I-J_{F P} \dot{\theta}_{F P}\right)
$$

where $J_{P I S}^{\#}$ is the pseudo-inverse of $J_{P I S}$ and $\dot{\theta}_{F P}$ is given by the controller for the optimal foot placement strategy.

\section{RESUlts}

We simulated the fall control procedure on an ASIMOlike humanoid using Webots[20]. The robot's environment is set up with four objects as shown in Fig. 1. The robot is pushed at its trunk CoM with horizontal forces of different magnitudes and directions and the performance of safe fall controller for each case was analyzed. All forces are of duration $100 \mathrm{~ms}$. Some of the results are presented here.

Fig. 1 shows the safe fall regions and the scores corresponding to each evaluated foot placement strategy when pushed with a forward force of $230 \mathrm{~N}$. It also shows safe fall as a result of choosing Take a Step strategy after identifying that No Action and Lift a Leg do not result in a safe fall. When the robot is pushed with a backward force of $210 \mathrm{~N}$, the default fall is safe. Our planning procedure successfully detects steady fall and chooses No Action as the best strategy, which results in a safe fall as shown in Fig. 6. Fig. 7 shows the safe fall behavior as a result of choosing Lift a Leg strategy after identifying a steady fall when pushed with a forward force of $210 \mathrm{~N}$.

Fig. 8 shows safe fall as a result of choosing Take a Step and Partial Inertia Shaping. As expected we can see significant arm motions in this case. Although the push force is in the same direction as in Fig. 7, the robot falls in a different direction due to high nonlinearity in the system. Fig. 9 shows safe fall as a result of performing Whole Body Inertia Shaping when all foot placement strategies fail to produce safe fall and No Action strategy is the optimal foot 


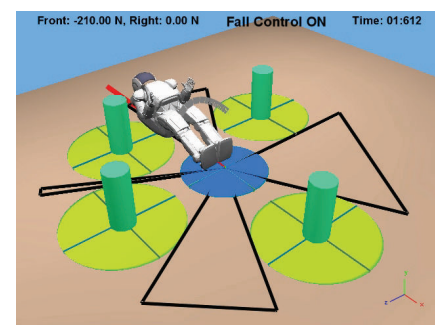

Fig. 6. Successful detection of safe fall with no support polygon change. The robot was pushed with a backward force of $210 \mathrm{~N}$, duration $100 \mathrm{~ms}$.

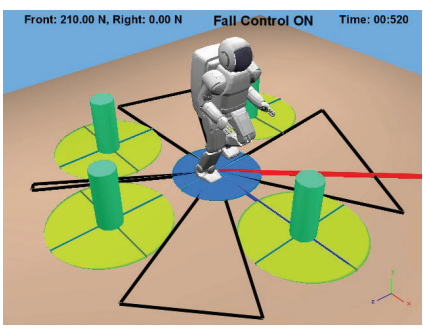

(a)

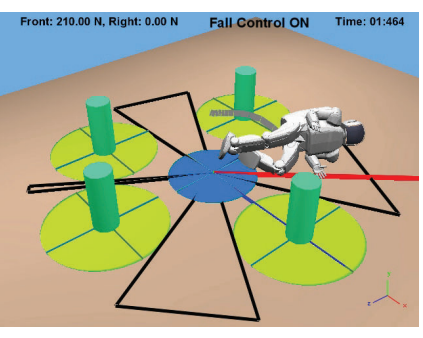

(b)
Fig. 7. (a) Lifting a leg to change direction, and (b) Safe fall as a result. The robot was pushed with a forward force of $210 \mathrm{~N}$, duration $100 \mathrm{~ms}$.

placement strategy, in the sense it is the best of all the unsuccessful strategies.

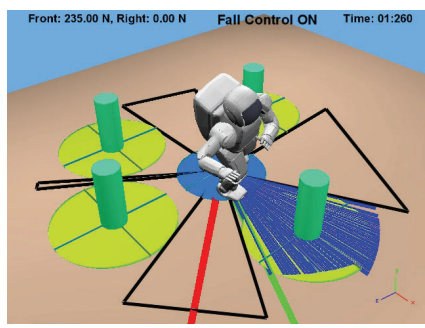

(a)

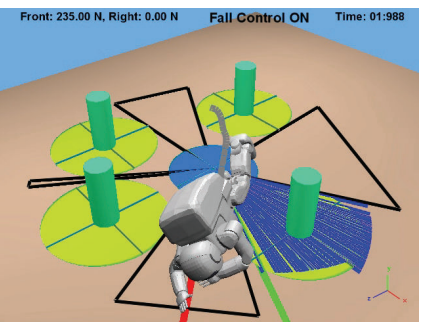

(b)
Fig. 8. (a) Taking a step and moving arms to perform partial inertia shaping, (b) Safe fall as a result of Take a Step and partial inertia shaping Strategies. The robot was pushed with a forward force of $235 \mathrm{~N}$ for $100 \mathrm{~ms}$. The green line shows the unsafe fall direction if partial inertia shaping was not used.

In order to emphasize the significance of partial inertia shaping, we would like to compare the outcomes of different strategy executions for the same case. In Fig. 10, we compare No Action, Take a Step and partial inertia shaping strategies when the robot was pushed with a forward force of $235 \mathrm{~N}$. The CoM trajectories show that safe fall was produced by using partial inertia shaping with Take a Step strategy.

All the above results are for cases where the robot was standing stationary when pushed. We also tested the fall control strategy for cases where the robot was pushed during walking. One result is shown in Fig. 11.

Full simulations of all the above results can be found in the companion video "Generalized Direction Changing Fall Control of Humanoid Robots Among Multiple Objects".

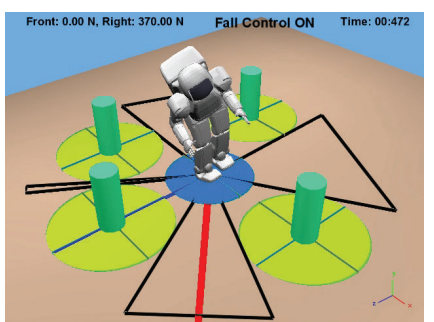

(a)

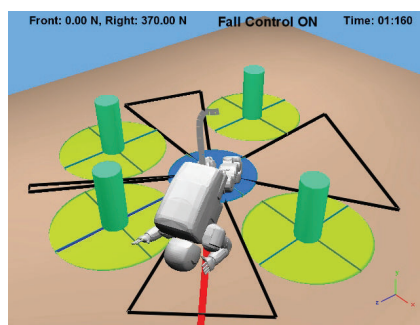

(b)
Fig. 9. (a) Whole body inertia shaping starts when no foot placement strategy produces safe fall and No Action is optimal, and (b) Safe fall as a result. The robot was pushed with a force of $370 \mathrm{~N}$ for $100 \mathrm{~ms}$, to the right.

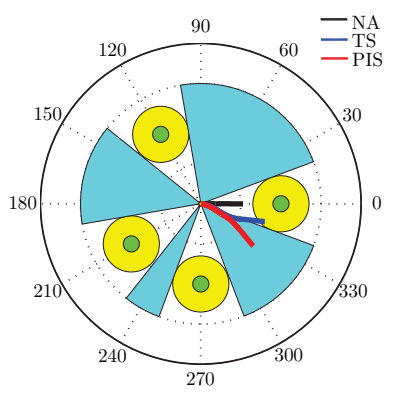

Fig. 10. Comparing the performances of different strategies. NA - No Action, TS - Take a Step, and PIS - Take a Step + Partial Inertia Shaping. The CoM trajectory during each strategy execution is shown. The robot is pushed with a forward force of 235 N. Only PIS produces safe fall.

\section{CONClusions And Future Work}

We presented a generalized planning and control algorithm for direction changing fall control of humanoid robots among multiple objects. The planner assigns scores and selects a desired fall direction. Next it logically evaluates a set of strategies and selects the optimal strategy for the desired direction. The controller executes this strategy in an interactive manner such that real-time modifications can be made in case there is a risk of failure.

In this work we have introduced the concept of control trigger and distinguished it from fall trigger, which was used in earlier work. While fall trigger corresponds to the mere prediction of a fall, control trigger corresponds to when the robot can make a useful control decision.

We presented partial inertia shaping, an inertia shaping technique that uses only a subset of the humanoid joints. Partial inertia shaping can be performed simultaneously with foot placement to improve the fall performance. The controller employed whole body inertia shaping when other strategies were predicted to fail or when the selected strategy was sensed to leading to failure. Several successful safe fall behavior of an ASIMO-like humanoid under a variety of external disturbances are reported.

The planning procedure presented in this paper assumes the following: $(i)$ the estimated fall directions are good approximations of the real fall directions and (ii) all strategy implementations are complete, by which we mean that the robot reaches the desired configuration corresponding to the strategy before it falls. The first assumption is due to the use 


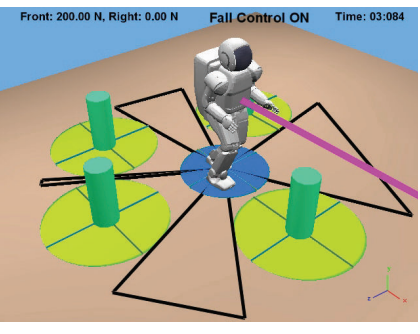

(a)

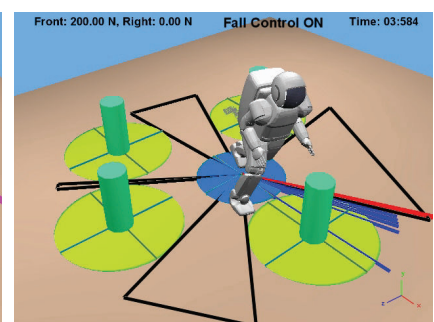

(b)

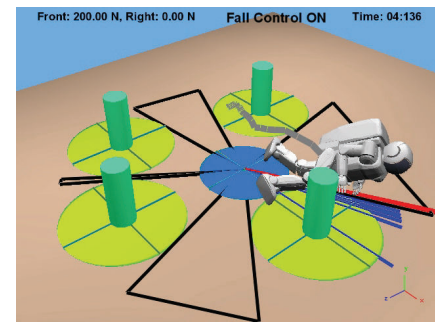

(c)

Fig. 11. (a) The robot is pushed with a $200 \mathrm{~N}$ forward force while walking, (b) Take a Step was the optimal strategy. This stepping location is different from the step it was about to take while walking. (c) Resulting safe fall.

of inverted pendulum model for estimating fall directions. Using more sophisticated models for better prediction of terminal fall direction is one of the future directions to be explored. Estimating error cones for robustness to each fall direction depending on its initial state is another possibility.

The second assumption has some effects in the performance of the safe fall controller. An incomplete strategy occurs only with the stepping strategy and it refers to the act of the controller unable to move the foot to its desired location before hitting the ground. An incomplete optimal plan can in no way be compared to other plans, and can result in complete failure.

While hardware experiment is our ultimate goal, additional future work regarding robustness estimation of our algorithms must be conducted first. Another interesting avenue is the effect of foot shape on the falling motion of humanoid.

\section{ACKNOWLEDGEMENTS}

Umashankar Nagarajan was supported by the summer internship program of Honda Research Institute, USA.

\section{REFERENCES}

[1] S-K. Yun, A. Goswami, and Y. Sakagami, "Safe fall: Humanoid robot fall direction change through intelligent stepping and inertia shaping", in IEEE Intn'l Conf. on Robotics and Automation (ICRA), May 2009, pp. 781-787.

[2] S-H. Lee and A. Goswami, "The reaction mass pendulum (RMP) model for humanoid robot gait and balance control", in Humanoid Robots, Ben Choi, Ed., pp. 167-186. In-Tech (www.intechweb.org), Austria, February, 2009.

[3] M. W. Walker and D. Orin, "Efficient dynamic computer simulation of robotic mechanisms", ASME Journal of Dynamic Systems, Measurement, and Control, vol. 104, pp. 205-211, Sept. 1982.

[4] K. Fujiwara, Kanehiro F., H. Saito, S. Kajita, K. Harada, and H. Hirukawa, "Falling motion control of a humanoid robot trained by virtual supplementary tests", in IEEE Intn'l Conf. on Robotics and Automation (ICRA), 2004, New Orleans, LA, USA, pp. 1077-1082.
[5] K. Fujiwara, S. Kajita, K. Harada, K. Kaneko, M. Morisawa, F. Kanehiro, S. Nakaoka, S. Harada, and H. Hirukawa, "Towards an optimal falling motion for a humanoid robot", in Humanoids06, 2006, pp. 524-529.

[6] K. Fujiwara, S. Kajita, K. Harada, K. Kaneko, M. Morisawa, F. Kanehiro, S. Nakaoka, S. Harada, and H. Hirukawa, "An optimal planning of falling motions of a humanoid robot", in IEEE/RSJ Intn'l Conf. on Intelligent Robots and Systems (IROS), 2007, pp. 456-462.

[7] K. Fujiwara, F. Kanehiro, S. Kajita, and H. Hirukawa, "Safe knee landing of a human-size humanoid robot while falling forward", in IEEE/RSJ Intn'l Conf. on Intelligent Robots and Systems (IROS), September 28- October 2 2004, Sendai, Japan, pp. 503-508.

[8] K. Fujiwara, F. Kanehiro, S. Kajita, K. Kaneko, K. Yokoi, and H. Hirukawa, "UKEMI: Falling motion control to minimize damage to biped humanoid robot", in IEEE/RSJ Intn'l Conf. on Intelligent Robots and Systems (IROS), September 30 - October 4, 2002 Lausanne, Switzerland, pp. 2521-2526.

[9] K. Ogata, K. Terada, and Y. Kuniyoshi, "Real-time selection and generation of fall damagae reduction actions for humanoid robots", in Humanoids08, Dec. -3 2008, Daejeon, Korea, pp. 233-238.

[10] K. Ogata, K. Terada, and Y. Kuniyoshi, "Falling motion control for humanoid robots while walking", in Humanoids07, Pittsburgh, 2007.

[11] R. Renner and S. Behnke, "Instability detection and fall avoidance for a humanoid using attitude sensors and reflexes", in IEEE/RSJ Intn'l Conf. on Intelligent Robots and Systems (IROS), Beijing, October 2006, pp. 2967-2973.

[12] J. G. Daniël Karssen and Martijn Wisse, "Fall detection in walking robots by multi-way principal component analysis", Robotica, vol. 27, no. 2, pp. 249-257, 2009.

[13] J. Ruiz del Solar, R. Palma-Amestoy, R. Marchant, I. ParraTsunekawa, and P. Zegers, "Learning to fall: Designing low damage fall sequences for humanoid soccer robots", Robotics and Autonomous Systems, vol. 57, no. 8, pp. 796-807, 2009.

[14] O. Höhn, J. Gačnik, and W. Gerth, Climbing and Walking Robots, chapter Detection and Classification of Posture Instabilities of Bipedal Robots, pp. 409-416, Springer Berlin Heidelberg, 2006.

[15] O. Höhn and W. Gerth, "Probabilistic balance monitoring for bipedal robots", in Intn'l J. of Robotics Research, 2009, vol. 28, pp. 245-256.

[16] T. Ishida, Y. Kuroki, and T. Takahashi, "Analysis of motions of a small biped entertainment robot", in IEEE/RSJ Intn'l Conf. on Intelligent Robots and Systems (IROS), September 28 - October 2, 2004 Sendai, Japan, pp. 142-147.

[17] A. Forner Cordero, Human gait, stumble and ... fall?, $\mathrm{PhD}$ thesis, University of Twente, Enschede, The Netherlands, 2003.

[18] S. M. LaValle, Planning Algorithms, Cambridge University Press, Cambridge, UK, 2006.

[19] J. Pratt, J. Carff, S. Drakunov, and A. Goswami, "Capture point: A step toward humanoid push recovery", in Humanoids06, December, Genoa, Italy 2006.

[20] Cyberbotics, "Webots: Professional mobile robot simulation", Intn' Journal of Advanced Robotic Systems, vol. 1, no. 1, pp. 39-42, 2004. 